ARTICLE

DOI: $10.1038 / s 41467-018-04721-8$

\title{
Large-scale gene losses underlie the genome evolution of parasitic plant Cuscuta australis
}

\author{
Guiling Sun 1,2, Yuxing Xu (1) 1,3,4, Hui Liu (1) 1, Ting Sun², Jingxiong Zhang (1) ', Christian Hettenhausen',

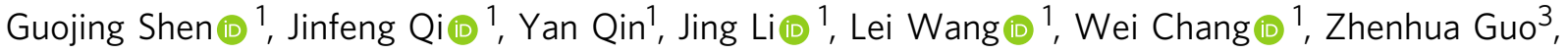 \\ Ian T. Baldwin (1D) ${ }^{5} \&$ Jianqiang Wu (D) ${ }^{1}$
}

Dodders (Cuscuta spp., Convolvulaceae) are root- and leafless parasitic plants. The physiology, ecology, and evolution of these obligate parasites are poorly understood. A highquality reference genome of Cuscuta australis was assembled. Our analyses reveal that Cuscuta experienced accelerated molecular evolution, and Cuscuta and the convolvulaceous morning glory (Ipomoea) shared a common whole-genome triplication event before their divergence. C. australis genome harbors 19,671 protein-coding genes, and importantly, $11.7 \%$ of the conserved orthologs in autotrophic plants are lost in C. australis. Many of these gene loss events likely result from its parasitic lifestyle and the massive changes of its body plan. Moreover, comparison of the gene expression patterns in Cuscuta prehaustoria/haustoria and various tissues of closely related autotrophic plants suggests that Cuscuta haustorium formation requires mostly genes normally involved in root development. The C. australis genome provides important resources for studying the evolution of parasitism, regressive evolution, and evo-devo in plant parasites.

\footnotetext{
${ }^{1}$ Department of Economic Plants and Biotechnology, Yunnan Key Laboratory for Wild Plant Resources, Chinese Academy of Sciences, Kunming 650201, China. ${ }^{2}$ Institute of Plant Stress Biology, State Key Laboratory of Cotton Biology, Department of Biology, Henan University, Kaifeng 475001, China. ${ }^{3}$ The Germplasm Bank of Wild Species, Kunming Institute of Botany, Chinese Academy of Sciences, Kunming 650201, China. ${ }^{4}$ University of Chinese Academy of Sciences, Beijing 100049, China. ${ }^{5}$ Max Planck Institute for Chemical Ecology, Jena 07745, Germany. These authors contributed equally: Guiling Sun, Yuxing Xu, Hui Liu. Correspondence and requests for materials should be addressed to J.W. (email: wujianqiang@mail.kib.ac.cn)
} 
A bout $1 \%$ of the flowering plants are haustorial parasites ${ }^{1}$, and some are responsible for severe yield losses in many crops. Although the evolutionary history of plant parasitism remains elusive, all parasitic plants, except the mycoheterotrophs, use a specialized haustorial organ to extract water and nutrients through vascular connections with the hosts ${ }^{2}$. The Cuscuta spp. (dodders) are typical obligate shoot parasites widely distributed worldwide comprising $~ 194$ species, and is the only genus of parasites in Convolvulaceae (Solanales). Cuscuta spp. exhibit massive changes of their body plans, being leaf- and rootless throughout their lifecycles (Fig. 1a). They contain trace amounts of chlorophyll, but cannot sustain themselves from their own photosynthesis. Although whether Cuscuta plants are hemior holoparasites remains debatable, they could be considered to be transitioning from hemiparasitism to holoparasitism. Through haustoria, dodders not only obtain water and nutrients, but secondary metabolites, mRNAs, and proteins from their host plants $^{3-5}$. These features make Cuscuta an important model to elucidate plant-parasite interactions and the evolution of plant parasites.

Here we sequence the genome of Cuscuta australis. Our analyses reveal that the genome of $C$. australis experienced massive gene losses, including important genes involved in leaf and root development, flowering-time control, as well as defense against pathogens and insects. Comparison of Cuscuta haustorium/prehaustorium gene expression patterns with the tissues from closely related autotrophic plants suggests that Cuscuta haustorium formation likely requires genes that are normally involved in root development.

\section{Results}

Genome assembly. The genome size of $C$. australis was estimated to be $272.57 \mathrm{Mb}$ from $k$-mer analysis. We next generated $26.6 \mathrm{~Gb}$ (97.6-fold coverage) of $C$. australis genome sequences from a single-molecule real-time (SMRT) sequencing platform. The $C$. australis genome sequence includes 249 contigs $(\mathrm{N} 50=3.63 \mathrm{Mb})$, and these contigs were further assembled to form 103 scaffolds $(\mathrm{N} 50=5.95 \mathrm{Mb}$; Supplementary Table 1). In total, $264.83 \mathrm{Mb}$ (219 contigs) of nuclear sequences $(97.16 \%$ of the estimated genome size) and $1.9 \mathrm{Mb}$ (30 contigs) of organellar sequences were acquired (Supplementary Table 2). The accuracy and heterozygosity were estimated to be $99.99 \%$ and $0.013 \%$, respectively (Supplementary Table 3). A total of $155 \mathrm{Mb}$ of repetitive elements were identified in the C. australis nuclear genome (Supplementary Table 4). Comparison with Ipomoea nil (Japanese morning glory; also Convolvulaceae), which is relatively closely related, indicated similar proportions of different types of repetitive elements between the two genomes (Supplementary Table 4), although the $I$. nil genome contains many more repeats (Supplementary Table 4), consistent with its larger genome size (734 $\mathrm{Mb}$ ). LTR (long terminal repeats) retrotransposons are the most dominant type of repeats in both $C$. australis and $I$. nil a

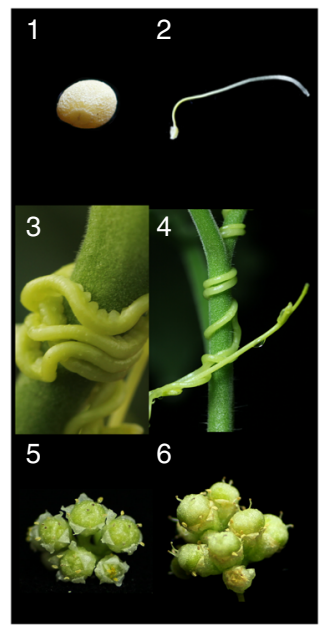

C

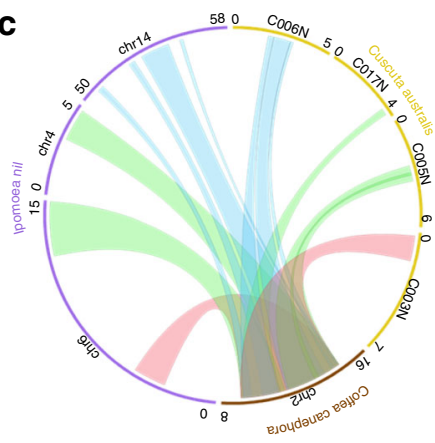

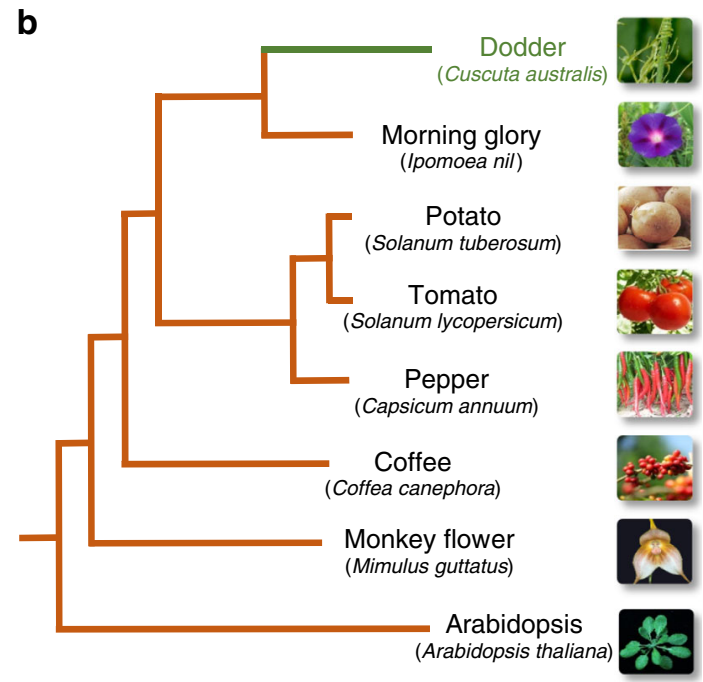

d

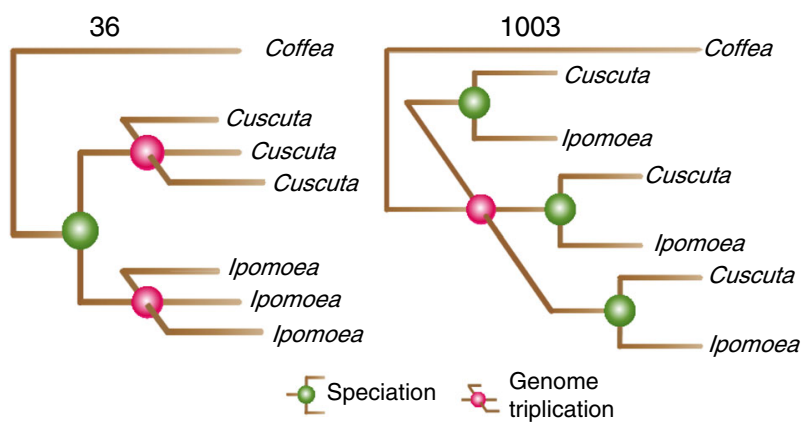

Fig. 1 Morphological traits and genome structure of $C$. australis. a Photographs of $C$. australis seed (1), seedling (2), vines twining around the wild tomato Solanum pennellii ( $3 \& 4$; partial haustoria can be seen in 3), flowers (5), and seed capsules (6). b Phylogenetic tree generated from genome-wide one-toone orthogroups (bootstrap values for all clades are 100\%). c Circos plot of a set of syntenic genome segments of $C$. australis, Japanese morning glory, and coffee. Numbers besides terminals of each karyotype denote the start and end of chromosome segment or contigs with unit of Mb. $\mathbf{d}$ Numbers of gene clades (shown on top of the trees) supporting different hypotheses on the order of speciation and whole-genome triplication event in the Cuscuta and Ipomoea lineage 
a

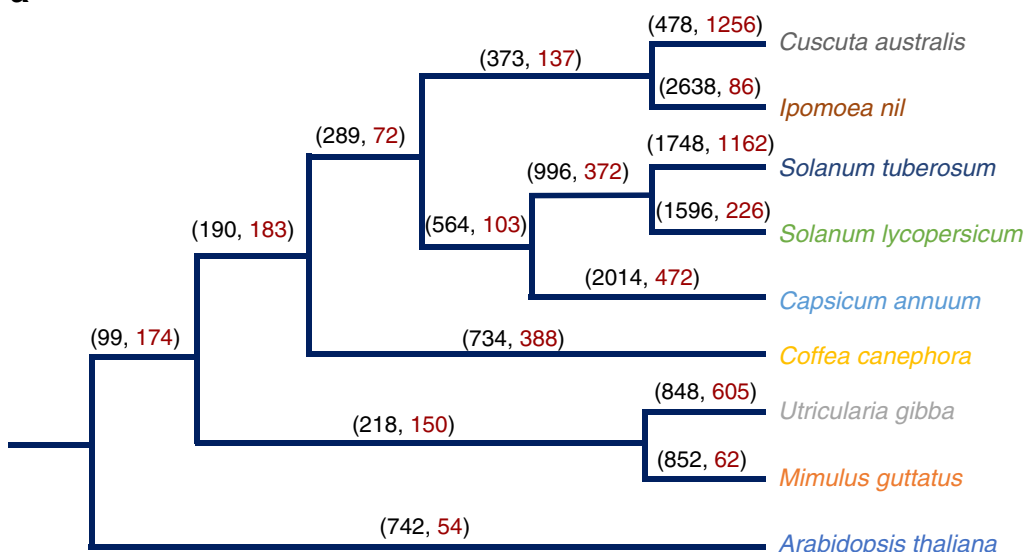

b

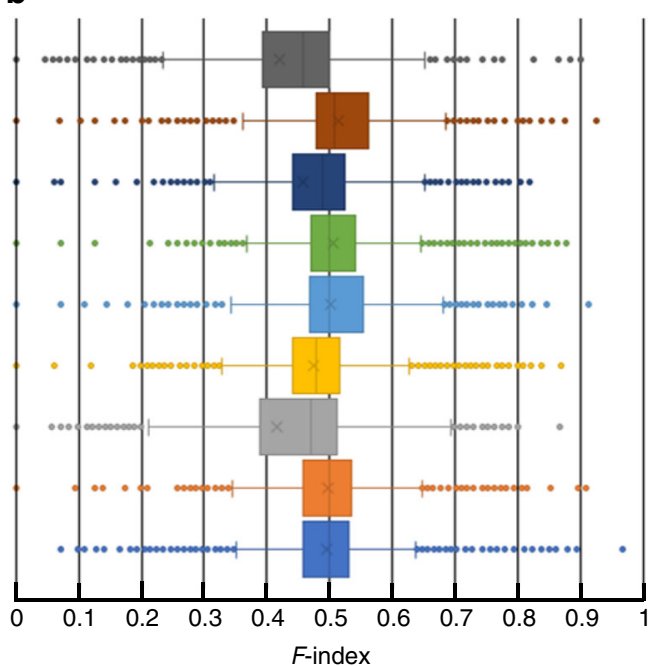

Fig. 2 Expansion and contraction in C. australis gene families. a Significantly expanded and contracted gene families. Brackets above each branch indicate numbers of expanded (in black, before comma) and contracted (in red, after comma) gene families. $\mathbf{b}$ Tukey boxplot overview of the differences among the gene numbers of the conserved gene families, based on the $F$-index values ( $F$-indices range from 0 to 1 ; when $F=,<$, or $>0.5$, the gene number in the given gene family is equal to, smaller, or greater than the average size of this gene family in all species). The left and right sides of the boxes are the first and third quartiles, respectively; means and medians of the data are shown as an " $x$ " and the bands in the boxes, respectively; for each box, the whiskers represent the smallest and biggest datum that are still within 1.5 times interquartile range of the lower and upper quartile, and the outliers are shown as dots

(Supplementary Table 4), and we specifically inspected the LTR/ Gypsy and LTR/Copia superfamilies. These two genomes exhibit large differences in LTR/Gypsy (84,083 in $I$. nil and 24,453 in C. australis) and LTR/Copia (96,355 in I. nil and 43,853 in C. australis) copy numbers; moreover, the sequences of $L T R / G y p s y$ and LTR/Copia members have also diverged as revealed by phylogenetic analysis (Supplementary Fig. 1).

Based on de novo gene structure prediction, homology comparison, and transcript data of both $C$. australis and the closely related relative C. pentagona ${ }^{6}, 19671$ genes could be annotated.

Phylogeny analysis. The phylogenetic position of Cuscuta was determined using 1796 one-to-one orthogroups (Supplementary Data 1b) identified from C. australis, Arabidopsis thaliana, and six lamiids plants-the Solanales Ipomoea nil, Solanum tuberosum (potato), Solanum lycopersicum (tomato), and Capsicum annuum (pepper), the Gentianales Coffea canephora (coffee), and the Lamiales Mimulus guttatus (monkey flower) (for simplicity, these seven autotrophic species are collectively named 7RefSpecies). Consistent with their phylogenetic relationship, Cuscuta forms a sister group with Ipomoea, and we estimated that these two lineages split $\sim 33$ million years ago (Supplementary Fig. 2). Notably, Cuscuta shows a much longer branch than does Ipomoea (Fig. 1b), providing genome-wide evidence consistent with the hypothesis that parasitic plants evolve rapidly ${ }^{7}, 8$. This result is statistically significant by both two cluster analysis and relative rate test (Supplementary Table 5 and Supplementary Table 6). Previously, a whole-genome duplication (WGD) event was detected in Ipomoea 9 . The syntenic blocks and trees of the syntenic gene groups of Cuscuta and Ipomoea vs. Coffea genome indicate that Cuscuta and Ipomoea experienced a whole-genome triplication event before their divergence from a common ancestor (Fig. 1c, d, Supplementary Fig. 3 to 5, Supplementary Table 7).

Contractions and expansions of gene families. Parasitism and large changes of body plan in Cuscuta suggest that many gene families might have experienced substantial alterations in sizes, including those that function in leaf and root physiology. To study gene family expansion and contraction, a rigorous bioinformatic pipeline was adopted to identify gene families (details see Supplementary Note 5). In addition, the genome of Utricularia gibba (Lentibulariaceae; an aquatic carnivorous bladderwort plant) was included in the analysis, given that $U$. gibba also exhibits large changes in body plan (e.g., no true roots). We identified a total of 13981 gene families in C. australis, U. gibba, and the 7Ref-Species (Supplementary Data 1a); among these, 1256 and 478 families in C. australis and 605 and 848 families in $U$. gibba were found to have had significant contractions and expansions, respectively, revealed by a maximum-likelihood analysis (Fig. 2a; Supplementary Data 1a). Moreover, box plots of the F-indices (details see Supplementary Note 5), which describe the differences among the gene numbers of the conserved gene families in the 7Ref-Species (namely, in Arabidopsis and at least five of the six remaining autotrophic species), indicate that in C. australis and U. gibba, gene numbers in $72 \%$ and $62 \%$ of the conserved gene families are below the averages, respectively (Fig. 2b).

Overall gene losses. The drastic contractions of gene families in C. australis and $U$. gibba suggest considerable gene losses in their genomes. Next, BUSCO analysis ${ }^{10}$ was carried out to map the 1440 conserved orthologs in land plants to the genomes of 7RefSpecies, C. australis, and U. gibba. Consistent with their contracted gene families, the missing BUSCOs in C. australis and $U$. gibba (16.30\% and $13.70 \%$, respectively) are more than those in the 7Ref-Species (1.40\% to $8.50 \%)$ (Supplementary Table 8 ).

To identify specific orthologous genes that are lost in $C$. australis and $U$. gibba, we developed a stringent genome-wide analysis pipeline to divide each gene family into small orthogroups using a method combining phylogenetic and syntenic analysis (details see Supplementary Note 6) and the functional annotations were assigned using Arabidopsis genome as the reference (Supplementary Fig. 6). This analysis resulted in 21487 orthogroups (Supplementary Data 1b). Among the 11995 
a

Ipomoea nil

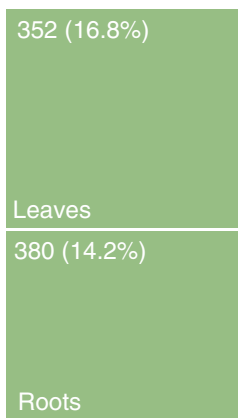

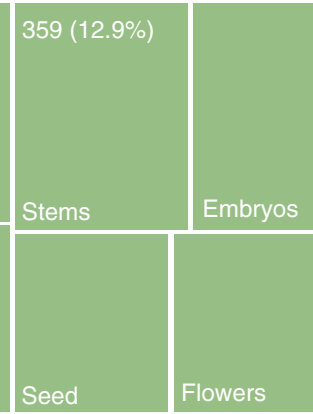

Solanum lycopersicum

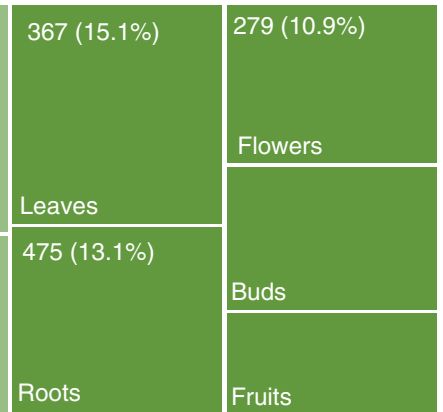

b

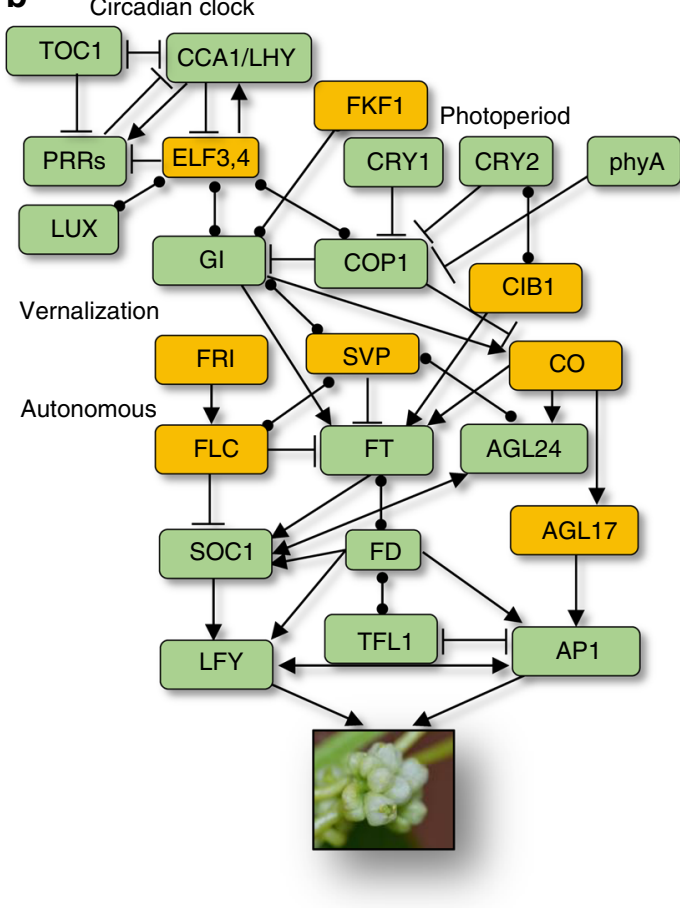

C

\begin{tabular}{|c|c|c|c|c|}
\hline & $R$ & TPS & $P 450$ & $R L K$ \\
\hline Cuscuta australis & 15 & 9 & 89 & 339 \\
\hline Ipomoea nil & 148 & 60 & 334 & 992 \\
\hline Solanum tuberosum & 390 & 31 & 410 & 655 \\
\hline Solanum lycopersicum & 264 & 34 & 255 & 685 \\
\hline Capsicum annuum & 311 & 53 & 455 & 701 \\
\hline Coffea canephora & 784 & 43 & 451 & 785 \\
\hline Mimulus guttatus & 336 & 53 & 346 & 720 \\
\hline Arabidopsis thaliana & 170 & 32 & 249 & 670 \\
\hline
\end{tabular}

Fig. 3 Gene losses in C. australis. a The principally expressed tissues (PETs) of the respective orthologs of C. australis lost genes in S. lycopersicum and I. nil. The boxes represent different tissues. The respective PETs of the orthogroups, which have no C. australis members, were identified in S. lycopersicum and $I$. nil. The numbers of orthogroups, which have PETs in leaves, roots, flowers, and other tissues, are shown in the boxes, and the respective percentages (proportional to the areas of the boxes) indicate the ratios between these indicated numbers and the numbers of all orthogroups whose PETs were identified to be the corresponding tissues of $I$. nil and S. Iycopersicum. b Simplified gene network controlling flowering time. Genes in green boxes are retained in C. australis, and the lost ones are in orange boxes. Arrows and T-ends represent promoting and inhibiting genetic interactions, respectively, and round dots at both ends symbolize genetic interactions with unknown directions. c Numbers of genes in the gene families of $R$ genes, TPSs, P450s, and RLKs

conserved orthogroups in the 7Ref-Species, there are 1402 and 1555 orthogroups whose C. australis and U. gibba members are absent, respectively (Supplementary Data $1 \mathrm{~b}$ and $1 \mathrm{c}$ ). Strikingly, 563 orthogroups have no C. australis and U. gibba orthologs, whose functions include phytohormone pathways, nutrient uptake, defense response, and root hair development (Supplementary Data 2a); 839 orthogroups specifically lost their members in C. australis, and these genes are mainly involved in response to light, photosynthesis, chloroplast RNA processing, and adventitious root development (Supplementary Data 2b); 992 orthogroups specifically have no members of $U$. gibba, and genes in these orthogroups are involved in signaling, response to stimuli, and protein modifications, among others (Supplementary Data 2 c). In addition to the lost genes, in C. australis genome we identified 1168 pseudogenes, which contain frame-shifts or premature stop codons. Among these, we found five flowering- time-related genes and 13 photosynthesis-related genes (Supplementary Data 3a).

We next inspected the tissue-specific expression patterns of the S. lycopersicum and I. nil (the closest autotrophs to Cuscuta among the 7Ref-Species) orthologs in the 1402 orthogroups, whose Cuscuta members are lost (Supplementary Data 1d). It was found that these orthologs' principally expressed tissues (the tissues, in which the expression levels of a given gene are at least 1 -fold greater than the averages of its expression levels in other tissues, are defined as the principally expressed tissues for that gene) are the leaves and root of both S. lycopersicum and I. nil (Fig. 3a). These data are consistent with the leaf- and rootless body plan of Cuscuta.

Loss of genes for leaf and root development. Next, the C. australis genome was specifically searched for genes that mediate leaf 
and root development, and it was found that a number of important genes involved in leaf and/or root development are absent (Supplementary Data 1c): The orthologs of (1) LCR (leaf shape and vein formation); (2) TRN1/LOP1 (leaf patterning and lateral root development); (3) PLT1, 2, and 5 (specification of root stem cell niche); (4) SMB (lateral root cap maturation); (5) all the CASP genes which are required for Casparian strip formation; (6) WAK1 to 5 (root cell expansion); (7) WOX3, 5, and 7 (embryonic patterning, stem cell maintenance, and organ formation).

Loss of genes for nutrient uptake. We found that many genes involved in potassium $(\mathrm{K})$, phosphate $(\mathrm{P})$, and nitrate $(\mathrm{N})$ uptake from soil are lost in C. australis. For $\mathrm{K}^{+}$uptake: the orthologs of HAK5 (a high affinity $\mathrm{K}^{+}$transporter), KAT3/AtKC1 (a general regulatory negatively modulating many inward Shaker $\mathrm{K}^{+}$ channels), and $\mathrm{CHX} 20\left(\mathrm{a} \mathrm{Na}^{+}\left(\mathrm{K}^{+}\right) / \mathrm{H}^{+}\right.$antiporter) are absent in C. australis. The following orthologs important for $\mathrm{P}$ uptake are also lost: $\mathrm{PHO} 2$ (P uptake and translocation), PHT2;1 (a chloroplast $\mathrm{P}$ transporter), and SPX3 (P signaling). Several genes involved in $\mathrm{N}$ uptake are missing as well, such as the orthologs of NAXT1 (a nitrate efflux transporter), and NLP7 (a positive regulator for nitrate-induced gene expression); moreover, while the low-affinity nitrate transporters NRT1.1 and 1.2 are retained, the high-affinity nitrate transporters NRT2.1 and 2.2 and NRT3.1 are absent.

Loss of photosynthesis genes. C. australis has very limited photosynthetic capability (Supplementary Fig. 7). Among 248 photosynthesis-related genes in Arabidopsis annotated by GO, 38 C. australis orthologs are missing (Supplementary Data 1c). The plastome of C. australis (Supplementary Fig. 8) appears to have gene contractions (81 genes remain, while the plastome of $I$. nil harbors 104 genes), and is similar to those of the previously sequenced Cuscuta species ${ }^{11,12}$ (Supplementary Data 4), especially C. obtusiflora and C. gronovii, all of these belong to the subgenus Grammica with C. australis. Notably, all these Cuscuta plastomes lack $n d h$ genes encoding proteins for forming the $\mathrm{NADH}$ dehydrogenase complex functioning in electron cycling around photosystem I under stress. These data concur with the generally accepted notion that $n d h$ genes are first to be lost in the initial stage in the evolution of parasitism, apparently due to relaxed selective constraint ${ }^{13}$ (Supplementary Data 4).

Loss of genes controlling flowering time. Leaves play a critical role in perceiving environmental signals and thereby modulate the physiology of their own as well as other plant parts, such as activating flowering ${ }^{14}$. We speculate that the leafless dodders may have unique means of regulating flowering time and many flowering-time-control genes may have been lost. A database of flowering-time gene networks in Arabidopsis was recently constructed $^{15}$. We found that among the 295 coding genes listed in this database, 26 are lost (Supplementary Data 1c), including the well-known FLC, FRI, SVP, AGL17, and CO (Fig. 3b). Moreover, the circadian clock genes ELF3 and 4, ARR3 and 4, and CDF1 and 3 are also missing. FKF1 and CIB1, which are essential in the photoperiod pathway, are also lost (Fig. 3b). Flowering time is controlled by multiple pathways ${ }^{16}$, and it appears that the vernalization, temperature, autonomous, circadian clock, and photoperiod pathway all seem to be nonfunctional (Fig. 3b). The regulation of $C$. australis flowering time is particularly interesting to explore further.

Loss of defense-related genes. Leaves and roots are the most common sites of pathogen and insect infestation. We speculate that after the ancestor of Cuscuta became leaf- and rootless, reduced exposure to pathogens and insects relaxed selective constraints on defense-related genes, resulting in their eventual loss. Specifically, four gene families were inspected in detail (Fig. 3c). $R$ (resistance) genes are the critical components of plant immunity. Even though there is a possibility that the ancestor of Convolvulaceae experienced a reduction of $R$ genes after the split between Convolvulaceae and other Solanales lineages, as I. nil has the smallest number of $R$ genes (148; Supplementary Data $3 b$ ) among the 7Ref-Species, the reduction of $R$ genes in Cuscuta is still drastic: $C$. australis genome harbors only $15 R$ genes (Fig. $3 c$ and Supplementary Data $3 b)$. Terpenes function as defenses against insects and pathogens. We predicted 9 terpene synthase genes (TPSs) in C. australis, and there are 31 to 60 TPSs in the 7Ref-Species (Supplementary Data 3c). Many P450 enzymes are involved in the biosynthesis of plant secondary metabolites, which are required for adaptation to biotic and abiotic stresses ${ }^{17}$. While more than 240 P450s are encoded by the genomes of the 7Ref-Species, C. australis genome harbors only 89 (Supplementary Data 3d). Receptor-like kinases (RLKs) are important in plant development and resistance to abiotic and biotic stresses. $C$. australis genome comprises only 339 RLK genes, much less than in the 7Ref-Species (at least 655 in potato) (Supplementary Data $3 e$ ). Consistently, several well-studied genes in plant resistance to pathogens are also absent in C. australis, including EDS1, EDS5, FMO1, SAG101, and PAD4, which are essential for plant resistance to diseases, and $A L D 1$, which is critical for systemic acquired resistance.

Origin of haustorium. The haustorium is a parasitic plantspecific organ, playing a critical role in establishing parasitism. $C$. australis and C. pentagona RNA-seq data ${ }^{6}$ were assembled using the C. australis genome as the reference (mapping ratios $96 \%$ and $75 \%$, respectively; Supplementary Table 9). In Cuscuta prehaustoria and haustoria, we identified 2466 principally expressed genes (PEGs; at least one-fold greater than the average of the expression levels in all the other tissues) belonging to 1299 orthogroups (Supplementary Data 1d, Supplementary Data 3f). GO analysis on these PEGs indicated enrichment of biological processes of metabolic process, transport, lignin and xyloglucan metabolism, and transcriptional regulation genes (Supplementary Data $2 \mathrm{~d}$ ). This is consistent with the haustorial function of transporting host substances and the findings that dynamic cell wall remodeling in parasite haustoria is important in the establishment of parasitism ${ }^{18-20}$. The biggest proportion of these 1299 corresponding orthologs' principally expressed tissues in S. lycopersicum and I. nil were found to be the roots (Supplementary Fig. 9, Supplementary Data 1d). These data imply that the evolution of Cuscuta haustorium may be related to expression changes of genes involved in root development. Similarly, comparative transcriptome analyses on three root parasites, Triphysaria versicolor, Striga hermonthica, and Phelipanche aegyptiaca, revealed that parasitism genes are derived primarily from root and floral tissues ${ }^{18}$.

Next, we performed a HYPHY analysis to obtain the genes that underwent positive selection and relaxed purifying selection after the divergence between the ancestors of Cuscuta and Ipomoea lineage, as these genes might be associated with the speciation of Cuscuta and evolution of parasitism. GO terms including "response to hormones", "DNA methylation", "regulation of transcription", and "cell wall-related metabolism" were enriched from the 1124 positively selected genes (Supplementary Data 3g, Supplementary Data 2e), and among these, 115 are principally expressed in prehaustoria/haustoria (Supplementary Data 3f), including a pectin esterase, receptor-like kinases, transcription factors, a serine carboxypeptidase, and transporters. We also 
found that 3890 genes (Supplementary Data 3h) exhibited signatures of relaxed purifying selection. The enriched GO terms include "terpenoid biosynthetic process", "nitrate assimilation", "photosystem II assembly", and "regulation of signal transduction" (Supplementary Data 2f), and 504 genes with relaxed purifying selection (Supplementary Data 3f) are principally expressed in prehaustoria/haustoria, such as genes encoding subtilisin-like proteases and an ABC transporter.

Gene family expansion, mainly caused by gene duplication, often leads to neofunctionalization among the gene family members and is thought to be an important driving force in the acquisition of novel phenotypes. Thus, we performed GO analysis on the 3099 expanded gene family members of $C$. australis (Supplementary Data 3i, Supplementary Data 2g). It was found that "response to auxin" and "DNA methylation" were enriched from the members of expanded gene families; among them, 109 genes are principally expressed in prehaustoria/ haustoria (Supplementary Data 3f). These data are consistent with the finding that many haustorial genes in Orobanchaceae parasites also experienced relaxed purifying and/or positive selection and may play an important role in the evolution of the parasitic lifestyle ${ }^{18}$.

Five positively selected genes from the expanded gene families were found to be principally expressed in haustoria (Supplementary Fig. 10. and Supplementary Data 3f). Among these, one encodes a putative $\alpha / \beta$-hydrolase highly similar to Nicotiana sylvestris DAD2/DWARF14 (84\% identity), which is the strigolactone receptor in autotrophic plants, implying that neofunctionalization of $\alpha / \beta$-hydrolase genes might also be involved in the parasitization process in Cuscuta, as they are in the root parasites Striga and Orobanche ${ }^{21}$.

\section{Discussion}

How plants evolved parasitism is still unclear. Our transcriptomic data and molecular evolution analysis suggest convergent evolution in Cuscuta and Orobanchaceae root parasites ${ }^{18}$. The haustorium of Cuscuta probably evolved from root tissues and that of Orobanchaceae parasites recruited genes normally involved in the development of root and floral tissues. Moreover, a relatively large fraction of the genes that experienced positive selection and/or relaxed selection are principally expressed in prehausotria/haustoria in both Cuscuta and Orobanchaceae, and these genes may be related to parasitization and/or evolution of parasitism.

Our comparative genomic analyses indicate that the C. australis genome experienced remarkably high levels of contraction, and this is consistent with Cuscuta's parasitic lifestyle and large changes in body plan-leaf- and rootless as well as intensive dependence on host-derived metabolites and signals. Given the importance of new genes that bring about novel phenotypes, it is possible that the autotrophic ancestor evolved the haustorium from root tissues through the neofunctionalization of duplicated genes and transcriptional reprogramming in the Cuscuta lineage. Cuscuta is transitioning from hemiparasitism to holoparasitism. Among the recognized major parasite lineages, three contain only hemiparasites, while eight are solely holoparasites ${ }^{1}$, implying that holoparasitism may have additional selective advantages over hemiparasitism. Hypothetically, the dramatic changes in body plans, including the degeneration of leaf and root could allow these parasites to reallocate carbon and nitrogen resources, which are required for their development and growth, to reproduction and could also enable the holoparasites to better adjust their physiology according to that of the hosts by eavesdropping on host signaling molecules.

Wicke et al. ${ }^{13}$ analyzed the plastomes of nonparasitic, hemiparasitic, and nonphotosynthetic parasitic plants in
Orobanchaceae, and found that the transition from autotrophic plants to obligate parasites relaxes functional constraints on plastid genes in a stepwise manner. Similar analyses could not be done in Cuscuta, as it is the only parasitic lineage in the Convolvulaceae. The large body plan changes that were associated with the parasitic lifestyle relaxed selective constraints on several core pathways, such as photosynthesis ${ }^{13}$, flowering-time control, root and leaf development, nutrient acquirement, and defense against pathogens and insects. Under relaxed selection pressure, some genes of these pathways were pseudogenized and melted into the background of surrounding DNA because of accumulation of recurrent mutations, or were even deleted from the genome, finally leading to gene losses. In C. australis genome, 1168 genes were identified as pseudogenes, although some may still be functional or even have gained new functions ${ }^{22}$. It is likely that the majority of these pseudogenes have degenerated or are in the process of being deleted, following the fate of the lost genes. Indeed, $C$. australis genes that have undergone relaxed purifying selection include those related to terpenoid biosynthetic process (defense), nitrate assimilation (nutrient acquirement), and photosystem II assembly (photosynthesis). Resequencing of other Cuscuta species may shed light on the recent gene loss events and the underlying mechanisms (e.g., transposon insertion, fragment deletion, and rapid accumulation of mutations) in Cuscuta.

Large scale gene loss is also evident from the genomic data of human parasites Pediculus humanus humanus (body louse) ${ }^{23}$, Trichinella spiralis (a zoonotic nematode) ${ }^{24}$, and Giardia lamblia (an intestinal protist) ${ }^{25}$, and from an Arabidopsis pathogen, the obligate biotrophic oomycete Hyaloperonospora arabidopsidis ${ }^{26}$. Here, we also detected that $U$. gibba also experienced a large number of gene loss events during its evolution, likely resulting from its body plan changes (mainly loss of root) and lifestyle alteration (carnivory). It would also be of importance to compare the genomes of Cuscuta and those of Orobanchaceae root parasites. We expect that Orobanchaceae parasites, especially holoparasites, such as Orobanche species, may also have a large number of lost nuclear genes, some of which may bear the same functions as those in Cuscuta, such as the genes that function in leaf development and photosynthesis. The C. australis genomic data strongly support the notion that regressive evolution, which is associated with extensive gene loss, is likely to be pervasive and adaptive during the evolution of holo- /obligate parasites ${ }^{27,} 28$. Comparative genomics between the genome data of $C$. australis and autotrophic plants provides an important resource for studying genome reduction, regressive evolution, parasitism, and evo-devo in plant parasites.

\section{Methods}

DNA sample preparation and sequencing. The seeds of C. australis were originally purchased from a Chinese traditional medicinal herbs store in Kunming, China, in 2011, and had been cultivated for five generations in a glasshouse at the Kunming Institute of Botany, Chinese Academy of Sciences. Voucher specimens of C. australis can be accessed at the Herbarium of the Kunming Institute of Botany, Chinese Academy of Sciences (accessions Nos. WJQ-001-1 and WJQ-001-3).

Seedlings were prepared from seeds of the fifth generation and were infested on soybean plants (for the germination procedure, see Li et al. ${ }^{29}$ ). DNA isolated from young vines collected from one individual $C$. australis was used for genomic library construction. Three genomic DNA libraries with 350-bp, 2-kb, or 5-kb insertions were constructed for Illumina sequencing. For PacBio sequencing, five DNA libraries with $20-\mathrm{kb}$ insertions were sequenced on a PacBio RS II instrument using the P6v2 polymerase binding and C4 chemistry kits (P6-C4). A total of $26.6 \mathrm{~Gb}$ from $1,953,966$ reads were obtained by processing 24 single-molecule real-time (SMRT) cells. The average and N50 of SMRT subread length were $9.6 \mathrm{~kb}$ and 13.6 $\mathrm{kb}$, respectively.

Transcriptome sample preparation and sequencing. C. australis tissues of seeds just-germinated seeds ( 1 day after imbibing), seedlings ( 5 days after imbibing), prehaustoria, haustoria, stems far from haustoria, stems near haustoria, flower buds, flowers, and seed capsules were collected and RNA samples were extracted 
from these tissues using the TRI Reagent (Sigma). Libraries were constructed for each tissue according to the TrueSeq RNA Sample Preparation protocol, and sequenced on an Illumina HiSeq-2500. Sequences are deposited in NCBI under BioProject PRJNA394036.

C. pentagona transcriptomic short reads dataset from Ranjan et al. ${ }^{6}$ were retrieved from the NCBI Short Read Archive under accession numbers SRR965929, SRR965963, SRR966236, SRR966405, SRR966412, SRR966513, SRR966542, SRR966549, SRR966619 to SRR966622, SRR967154, SRR967164, SRR967181 to SRR967190, SRR967275 to SRR967289, and SRR967291.

Genome survey. We used $23.29 \mathrm{~Gb}$ of HiSeq reads to estimate the genome features using the $\mathrm{GCE}^{30}$ (v1.0.0) software based on $k$-mer depth-frequency distribution. A total of 11,700,637,064 17-mers were counted. Given the unique $k$-mer depth of 42 , we calculated that the genome size $=$ KmerCount/Depth $=272.57 \mathrm{Mb}$. The repeat content was estimated to be $58.94 \%$ based on $k$-mer depth distribution.

De novo assembly. SMRT subreads were corrected, trimmed, and assembled using $\mathrm{CANU}^{31}$ (v1.3), a genome assembler built on the basis of Celera assembler. Briefly, SMRT subreads were firstly self-corrected based on an overlap-layout algorithm. Erroneous regions of error-corrected SMRT subreads were then trimmed to increase accuracy. We constructed an initial assembly using CANU with those trimmed error-corrected SMRT subreads following the parameters "genomeSize = $273 \mathrm{~m}$ errorRate $=0.025$ " and then used Quiver ${ }^{32}(\mathrm{v} 4.0)$ to generate consensus sequences by aligning SMRT subreads to correct the errors in the assembly. Lastly, we used Pilon ${ }^{33}$ (v1.18) to perform the second round of error correction with HiSeq reads from the 350-bp-insert library. The resulted error-corrected assembly was named version 1.0 and used in the subsequent analyses. A hierarchical method was also applied to concatenate adjacent contigs: $\operatorname{SSPACE}^{34}$ (v 3.0) was first used to build scaffolds using HiSeq data from the mate-pair libraries and the contigs built from the PacoBio data. N50 of the resulted scaffolds reached 5.9 Mb. SSPACELongRead $^{35}$ (v1-1) was further applied to build superscaffolds with PacBio long reads, nevertheless, no linking information between scaffolds were found and no improvements were acquired (Supplementary Table 1).

Assembly assessment. The accuracy and heterozygosity rate of the genome assembly were estimated using the following procedure: adaptors were removed from the short paired-end reads obtained from the 350-bp insert size library, and then the reads were aligned to the PacBio assembly, which had been corrected with Pilon $^{33}$ (v1.18), using bowtie2 $2^{36}$ (v 2.2.4) to generate a bam file. A total of $97.9 \%$ reads could be mapped. Samtools ${ }^{37}$ (v 1.3.1) were used to sort the bam file, and Freebayes $^{38}$ (v1.1.0) was applied to call variants. Compared with PacBio assembly as the reference genome, homozygous SNPs or indels were considered to be assembly errors, and heterozygous SNPs or indels were regarded as heterozygous sites. The accuracy was estimated to be $99.99 \%$ and heterozygosity was estimated to be $0.013 \%$ (Supplementary Table 3 ). Error rate of the genome assembly before Pilon correction was also estimated using the same set of methods. A total of 980 SNPs and 101825 Indels were corrected after polishing.

Data availability. The genome assembly, gene models, and sequence reads are available at the NCBI under the BioProject PRJNA394036, and the former two can also be accessed at http://www.dodderbase.org. Phylogenetic analysis data for detection of gene losses, gene alignments used for selection analysis, and pseudogene annotations can be accessed at https://doi.org/10.6084/m9.figshare.6072131. All data are also available from the corresponding author upon request.

Received: 8 August 2017 Accepted: 23 April 2018

Published online: 11 July 2018

\section{References}

1. Westwood, J. H., Yoder, J. I., Timko, M. P. \& dePamphilis, C. W. The evolution of parasitism in plants. Trends Plant. Sci. 15, 227-235 (2010)

2. Yoshida, S., Cui, S., Ichihashi, Y. \& Shirasu, K. The haustorium, a specialized invasive organ in parasitic plants. Annu. Rev. Plant. Biol. 67, 643-667 (2016).

3. Kim, G., LeBlanc, M. L., Wafula, E. K., Depamphilis, C. W. \& Westwood, J. H. Genomic-scale exchange of mRNA between a parasitic plant and its hosts. Science 345, 808-811 (2014).

4. Birschwilks, M., Haupt, S., Hofius, D. \& Neumann, S. Transfer of phloemmobile substances from the host plants to the holoparasite Cuscuta sp. J. Exp. Bot. 57, 911-921 (2006).

5. Smith, J. D., Woldemariam, M. G., Mescher, M. C., Jander, G. \& De Moraes, C. M. Glucosinolates from host plants influence growth of the parasitic plant Cuscuta gronovii and its susceptibility to aphid feeding. Plant Physiol. 172, 181-197 (2016).
6. Ranjan, A. et al. De novo assembly and characterization of the transcriptome of the parasitic weed dodder identifies genes associated with plant parasitism. Plant Physiol. 166, 1186-1199 (2014).

7. Estep, M. C., Gowda, B. S., Huang, K., Timko, M. P. \& Bennetzen, J. L. Genomic characterization for parasitic weeds of the genus Striga by sample sequence analysis. Plant Genome 5, 30-41 (2012).

8. Bromham, L., Cowman, P. F. \& Lanfear, R. Parasitic plants have increased rates of molecular evolution across all three genomes. BMC Evol. Biol. 13, 126 (2013).

9. Hoshino, A. et al. Genome sequence and analysis of the Japanese morning glory Ipomoea nil. Nat. Commun. 7, 13295 (2016).

10. Simao, F. A., Waterhouse, R. M., Ioannidis, P., Kriventseva, E. V. \& Zdobnov, E. M. BUSCO: assessing genome assembly and annotation completeness with single-copy orthologs. Bioinformatics 31, 3210-3212 (2015).

11. Funk, H. T., Berg, S., Krupinska, K., Maier, U. G. \& Krause, K. Complete DNA sequences of the plastid genomes of two parasitic flowering plant species, Cuscuta reflexa and Cuscuta gronovii. BMC Plant Biol. 7, 45 (2007).

12. McNeal, J. R., Arumugunathan, K., Kuehl, J. V., Boore, J. L. \& Depamphilis, C. W. Systematics and plastid genome evolution of the cryptically photosynthetic parasitic plant genus Cuscuta (Convolvulaceae). BMC Biol. 5, 55 (2007).

13. Wicke, S. et al. Mechanistic model of evolutionary rate variation en route to a nonphotosynthetic lifestyle in plants. Proc. Natl Acad. Sci. USA 113, 9045-9050 (2016).

14. Putterill, J. \& Varkonyi-Gasic, E. FT and florigen long-distance flowering control in plants. Curr. Opin. Plant. Biol. 33, 77-82 (2016).

15. Bouche, F., Lobet, G., Tocquin, P. \& Perilleux, C. FLOR-ID: an interactive database of flowering-time gene networks in Arabidopsis thaliana. Nucleic Acids Res. 44, D1167-D1171 (2016).

16. Romera-Branchat, M., Andres, F. \& Coupland, G. Flowering responses to seasonal cues: what's new? Curr. Opin. Plant. Biol. 21, 120-127 (2014).

17. Morant, M., Bak, S., Moller, B. L. \& Werck-Reichhart, D. Plant cytochromes P450: tools for pharmacology, plant protection and phytoremediation. Curr. Opin. Biotechnol. 14, 151-162 (2003).

18. Yang, Z. et al. Comparative transcriptome analyses reveal core parasitism genes and suggest gene duplication and repurposing as sources of structural novelty. Mol. Biol. Evol. 32, 767-790 (2014).

19. Johnsen, H. R. et al. Cell wall composition profiling of parasitic giant dodder (Cuscuta reflexa) and its hosts: a priori differences and induced changes. New Phytol. 207, 805-816 (2015).

20. Olsen, S. et al. Getting ready for host invasion: elevated expression and action of xyloglucan endotransglucosylases/hydrolases in developing haustoria of the holoparasitic angiosperm Cuscuta. J. Exp. Bot. 67, 695-708 (2016).

21. Conn, C. E. et al. Convergent evolution of strigolactone perception enabled host detection in parasitic plants. Science 349, 540-543 (2015).

22. Balakirev, E. S. \& Ayala, F. J. Pseudogenes: are they "junk" or functional DNA? Annu. Rev. Genet. 37, 123-151 (2003).

23. Kirkness, E. F. et al. Genome sequences of the human body louse and its primary endosymbiont provide insights into the permanent parasitic lifestyle. Proc. Natl.Acad. Sci. USA 107, 12168-12173 (2010).

24. Mitreva, M. et al. The draft genome of the parasitic nematode Trichinella spiralis. Nat. Genet. 43, 228-235 (2011).

25. Morrison, H. G. et al. Genomic minimalism in the early diverging intestinal parasite Giardia lamblia. Science 317, 1921-1926 (2007).

26. Baxter, L. et al. Signatures of adaptation to obligate biotrophy in the Hyaloperonospora arabidopsidis genome. Science 330, 1549-1551 (2010).

27. Albalat, R. \& Canestro, C. Evolution by gene loss. Nat. Rev. Genet. 17, 379-391 (2016).

28. Wolf, Y. I. \& Koonin, E. V. Genome reduction as the dominant mode of evolution. Bioessays 35, 829-837 (2013).

29. Li, J. et al. The parasitic plant Cuscuta australis Is highly insensitive to abscisic acid-induced suppression of hypocotyl elongation and seed germination. PLoS ONE10, e0135197 (2015).

30. Liu, B. et al. Estimation of genomic characteristics by analyzing k-mer frequency in de novo genome projects. Preprint at https://arxiv.org/abs/ 1308.2012 (2012)

31. Koren, S. et al. Canu: scalable and accurate long-read assembly via adaptive kmer weighting and repeat separation. Genome Res. 27, 722-736 (2017).

32. Chin, C. S. et al. Nonhybrid, finished microbial genome assemblies from longread SMRT sequencing data. Nat. Methods 10, 563-569 (2013).

33. Walker, B. J. et al. Pilon: an integrated tool for comprehensive microbial variant detection and genome assembly improvement. PLoS ONE 9, e112963 (2014).

34. Boetzer, M., Henkel, C. V., Jansen, H. J., Butler, D. \& Pirovano, W. Scaffolding pre-assembled contigs using SSPACE. Bioinformatics 27, 578-579 (2011).

35. Boetzer, M. \& Pirovano, W. SSPACE-LongRead: scaffolding bacterial draft genomes using long read sequence information. BMC Bioinformatics 15, 211 (2014). 
36. Langmead, B. \& Salzberg, S. L. Fast gapped-read alignment with Bowtie 2. Nat. Methods 9, 357-359 (2012).

37. Li, H. et al. The sequence alignment/map format and SAMtools. Bioinformatics 25, 2078-2079 (2009).

38. Garrison, E. \& Marth, G. Haplotype-based variant detection from short-read sequencing. Preprint at https://arxiv.org/abs/1207.3907 (2012).

\section{Acknowledgements}

This work was supported by the Strategic Priority Research Program of the Chinese Academy of Sciences (CAS) (No. XDB11050200; J.W.). The authors also thank Drs. Lei Wang (Institute of Botany, CAS), Hongtao Liu (Institute of Plant Physiology and Ecology, CAS), Jinyong Hu and Chengjun Zhang (Kunming Institute of Botany, CAS), and Zhaojun Ding (Shangdong University) for valuable comments.

\section{Author contributions}

J.W., G.S., and Y.X. designed and conceived research; T.S., C.H., G.S. prepared plant tissues; W.C. performed photosynthesis experiments; T.S., and Y.Q. prepared DNA samples; J.L. and J.Q. prepared RNA samples; Y.X., and H.L. performed genome assembly and genome annotation; Y.X., H.L, and T.S. analyzed data; J.W., G.S., Y.X., H.L., J.Z., L.W., I.T.B., and Z.G. wrote the paper. All authors read and approved the final manuscript.

\section{Additional information}

Supplementary Information accompanies this paper at https://doi.org/10.1038/s41467018-04721-8
Competing interests: The authors declare no competing interests.

Reprints and permission information is available online at http://npg.nature.com/ reprintsandpermissions/

Publisher's note: Springer Nature remains neutral with regard to jurisdictional claims in published maps and institutional affiliations.

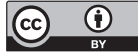

Open Access This article is licensed under a Creative Commons Attribution 4.0 International License, which permits use, sharing, adaptation, distribution and reproduction in any medium or format, as long as you give appropriate credit to the original author(s) and the source, provide a link to the Creative Commons license, and indicate if changes were made. The images or other third party material in this article are included in the article's Creative Commons license, unless indicated otherwise in a credit line to the material. If material is not included in the article's Creative Commons license and your intended use is not permitted by statutory regulation or exceeds the permitted use, you will need to obtain permission directly from the copyright holder. To view a copy of this license, visit http://creativecommons.org/ licenses/by/4.0/.

(C) The Author(s) 2018 